\title{
FISIOTERAPIA EM UNIDADES DE EMERGÊNCIA: UMA REVISÃO SISTEMÁTICA
}

\section{PHYSICAL THERAPY IN EMERGENCY DEPARTMENTS: A SISTEMATIC REVIEW}

\section{André Luiz Cordeiro', Tiane Greice Lima ${ }^{2}$}

Autor para correspondência: André Luiz Lisboa Cordeiro - andrelisboacordeiro@gmail.com 'Fisioterapeuta. Professor na Faculdade Nobre. Feira de Santana, Bahia, Brasil. ${ }^{2}$ Fisioterapeuta. Pós-graduada em Fisioterapia Hospitalar pela Escola Bahiana de Medicina e Saúde Pública.

RESUMO | Introdução: A emergência é o ambiente de entrada do paciente crítico ou potencialmente crítico na rede hospitalar. O fisioterapeuta, por muito tempo, foi um prestador de assistência para a equipe da emergência; porém, hoje, em alguns serviços, ele é componente fundamental dessa equipe. $A$ inserção do fisioterapeuta nas equipes de emergência ainda não está consolidada dependendo da necessidade de cada serviço. Objetivo: Descrever o papel do fisioterapeuta nas unidades de emergência (UE). Metodologia: Tratouse de uma revisão sistemática, onde foram analisados seis artigos científicos. Foram utilizados como referências, publicações nas línguas inglesas, espanhola e portuguesa, contidas nas seguintes bases de dados eletrônicos: MEDLINE (Pubmed), LILACS e SciELO. Resultados: 0 fisioterapeuta emprega todos os métodos e técnicas disponíveis, dentro da sua área de atuação, no processo de melhora do paciente e análise dos resultados obtidos na terapêutica. A influência da inserção do fisioterapeuta nas UE sobre o tempo de internação hospitalar em pacientes com lesões musculoesqueléticas periféricas, agravos cardiovasculares e respiratórios mostra uma redução do tempo de permanência desses pacientes sem presença de efeitos adversos. Porém, existe ainda uma incerteza sobre a regulamentação e são necessárias ações políticas e educacionais para esclarecer e apoiar esta área de prática clínica, baseado em evidencias clínicas apropriadas. Conclusão: A abordagem fisioterapêutica em UE está associada com diminuição da necessidade de internação hospitalar, aumento da satisfação do paciente e diminuição do quadro álgico.

\begin{abstract}
I Introduction: Emergency is the critical or potentially critical patient input environment in the hospital network. The physiotherapist, for a long time, was a caregiver for the emergency team; But today, in some services, it is a fundamental component of this team. The insertion of the physiotherapist in the emergency teams is not yet consolidated depending on the need for each service. Objective: To describe the role of physiotherapist in emergency units (UE). Methodology: It was a systematic review, where six scientific articles were analyzed. English, Spanish and Portuguese publications were used as references in the following electronic databases: MEDLINE (Pubmed), LILACS and SciELO. Results: The physiotherapist employs all available methods and techniques, within his / her area of action, in the process of patient improvement and analysis of the results obtained in the therapy. The influence of the insertion of the physiotherapist in the EU on the length of hospital stay in patients with peripheral musculoskeletal injuries, cardiovascular and respiratory disorders shows a reduction in the length of stay of these patients without adverse effects. However, there is still uncertainty about regulation and political and educational actions are needed to clarify and support this area of clinical practice, based on appropriate clinical evidence. Conclusion: The physiotherapeutic approach in the EU is associated with a reduction in the need for hospitalization, an increase in patient satisfaction and a decrease in pain.
\end{abstract}

Keywords: Physical Therapy Specialty; Emergencies; Ambulatory Care.

Palavras-chave: Fisioterapia; Emergências; Assistência ambulatorial. 


\section{INTRODUÇÃO}

A emergência é 0 ambiente de entrada do paciente crítico ou potencialmente crítico na rede hospitalar ${ }^{1}$. O fisioterapeuta, por muito tempo, foi um prestador de assistência para a equipe da emergência; porém, hoje, em alguns serviços, é componente fundamental dessa equipe ${ }^{2}$. O fisioterapeuta que atua na Unidade de Emergência (UE) tem como função prestar assistência ao paciente, avaliar, identificar os que possuem maior risco de complicações e grau de funcionalidade, executar tratamento das morbidades, promover a saúde e bem-estar social. Atualmente no Brasil, o principal objetivo do atendimento fisioterapêutico na UE é suporte rápido e eficiente para disfunções cardiorrespiratórias, evitando, assim, um possível agravamento no quadro clínico, como a necessidade de intubação orotraqueal, utilização de ventilação mecânica invasiva e evolução para a Unidade de Terapia Intensiva (UTI) ${ }^{3,4}$.

Esse tema torna-se importante pela possibilidade de inserção do fisioterapeuta em um campo ainda pouco explorado. Sendo que em muitos hospitais públicos do Brasil, a escassez de leitos de terapia intensiva propicia que muitos pacientes evoluam com piora do quadro ainda no serviço de emergência, portanto a presença do fisioterapeuta nesta unidade pode proporcionar uma otimização do quadro clínico do paciente: na descompensação respiratória, cardiovascular e músculo-esquelética, diminuindo assim a necessidade de internação hospitalar, reduzindo custos hospitalares e reinserindo precocemente $o$ paciente na sociedade ${ }^{5,6}$.

Contudo a atuação dos Fisioterapeutas nas UE dos hospitais, ainda não está consolidada ou definida pelos gestores atuais. Porém, principalmente, no Reino Unido e na Austrália, a discussão sobre a inserção dos Fisioterapeutas nas UEs tem promovido o questionamento e a investigação dos benefícios e dos espaços de atuação destes profissionais ${ }^{2,7}$.

Portanto o objetivo principal desse trabalho foi reunir da literatura dados sobre o impacto da abordagem fisioterapêutica nos pacientes admitidos em unidades de emergência.

\section{MATERIAL E MÉTODOS}

Trata-se de uma revisão sistemática realizada através de busca nas bases de dados MEDLINE (PubMed), LILACS e SciELO, em que os critérios de inclusão dos artigos selecionado consistiam em artigos nos idiomas inglês, espanhol e português, publicados no período de 2000 a 2017, que abordaram intervenções do fisioterapeuta na UE. As palavras chave utilizadas foram: "emergência" and "departamento de emergência" and "fisioterapia" and "hospital".

\section{Os critérios de seleção}

Artigos originais, que tratassem da assistência fisioterapêutica com abordagem cardiorrespiratória e/ou musculoesquelética nas UE. Foram excluídos os estudos que envolviam assistências em crianças e neonatos, estudos não randomizados, relatos de casos, observações clínicas e revisões.

\section{Extração dos dados}

Inicialmente, a seleção de estudos para extrair os dados necessários baseou-se na verificação de títulos de estudos, bem como na análise dos resumos disponíveis. Subsequentemente, os relatórios completos do estudo foram comparados com os critérios de inclusão pré-estabelecidos para determinar sua relevância para a revisão sistemática. Dois revisores, de forma independente e cega, atendendo aos critérios de inclusão e exclusão definidos no protocolo de pesquisa, extraíram os dados para examinar as características do estudo, os pacientes e a possível eficácia dos protocolos terapêuticos.

Para avaliar a qualidade metodológica do trabalho, utilizou-se a escala PEDro ${ }^{8}$, ferramenta que quantifica a qualidade de ensaios clínicos randomizados ou estudos quase-randomizados. A escala PEDro consisti em 11 itens. Um item na escala PEDro (critérios de elegibilidade) está relacionado à validade externa e geralmente não é usado para calcular a pontuação do método, deixando uma pontuação de 0 a 10 . Esta revisão sistemática foi concluída de acordo com as diretrizes de Relatórios 


\section{RESULTADOS}

Após análise por dois pesquisadores, dos 37 estudos inicialmente identificados, 31 artigos foram excluídos, incluindo estudos desenvolvidos em diferentes contextos clínicos, aqueles que não utilizaram a fisioterapia no cenário pesquisado ou não apresentaram um desenho metodológico apropriado. O fluxograma mostrado na Figura 1 aponta para os detalhes finais da seleção. Após análise detalhada para $\circ$ desenvolvimento desta revisão, sete estudos clínicos foram potencialmente selecionados, incluindo os critérios metodológicos estipulados para o resultado pretendido.

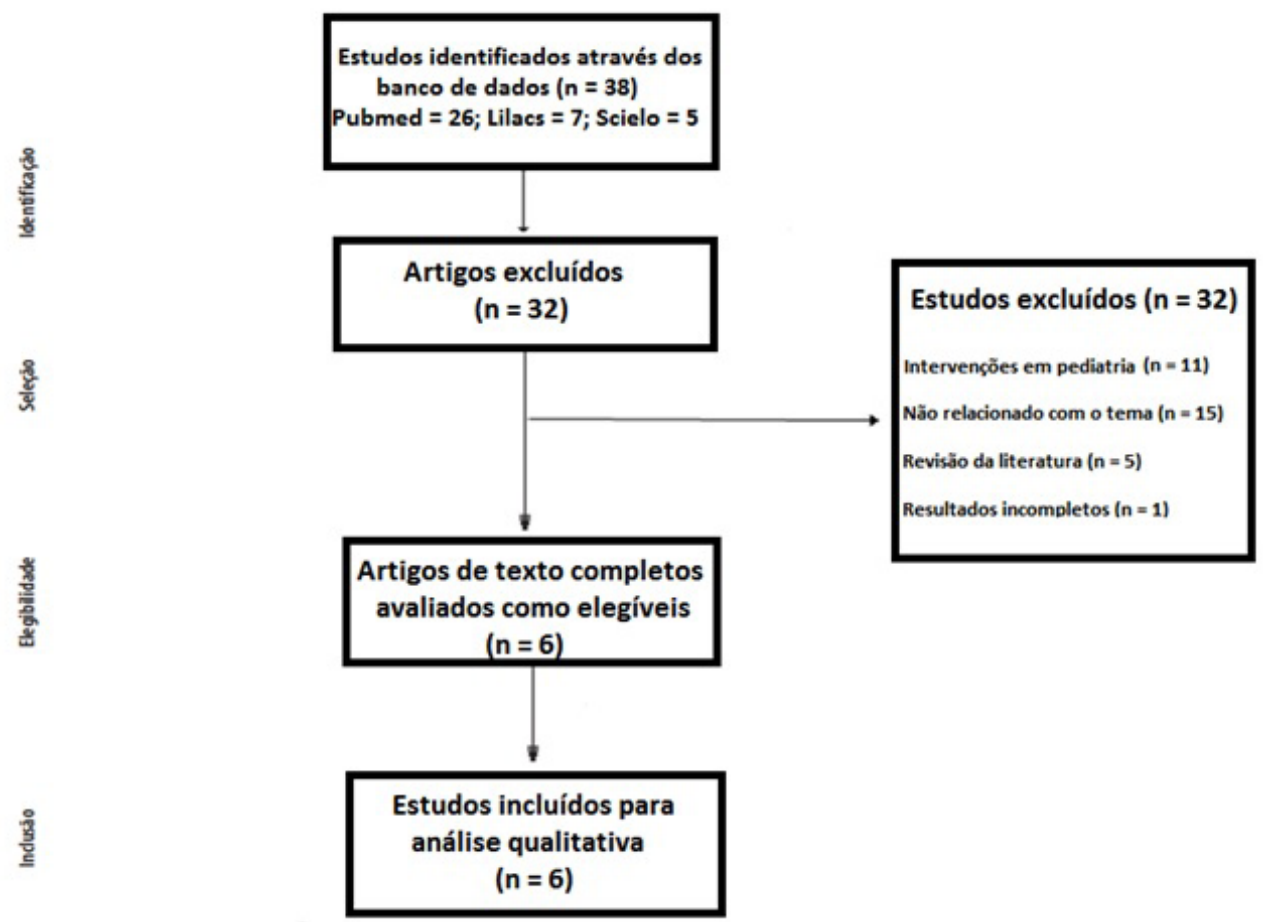

Figura 1. Fluxograma para obtenção dos ensaios clínicos randomizados no período de janeiro a março de 2017.

A qualidade metodológica avaliada através da escala PEDro é mostrada na tabela 1. Os seis estudos inclú́dos nesta revisão da literatura discutem o impacto da fisioterapia em pacientes admitidos em unidades de emergência. O tamanho da amostra variou de noventa a setecentos e sessenta e seis pacientes de ambos os gêneros atendidos nas unidades hospitalares.

Tabela 1. Estratificação de Ensaio clínico randomizado sobre fisioterapia em unidades de emergência através da Escala PEDro de janeiro a março de 2017.

\begin{tabular}{ccc}
\hline Autor & Local do Estudo & Escala PEDro \\
\hline Calthorpe et al7. & $\begin{array}{c}\text { Melbourne, } \\
\text { Austrália }\end{array}$ & $04 / 10$ \\
Taylor et al ${ }^{8}$. & Nova Zelândia & $05 / 10$ \\
Courtney et $\mathbf{a l}^{9}$. & Brisbane, Austrália & $04 / 10$ \\
Richardson et al ${ }^{10}$. & Reino Unido & $05 / 10$ \\
Jesudason et al ${ }^{11}$. & Adelaide, Austrália & $06 / 10$ \\
Lau et al ${ }^{12}$. & Hong Kong, China & $04 / 10$ \\
\hline
\end{tabular}


Dos seis estudos, dois demonstraram aumento significativo em relação à aplicação satisfação do paciente como melhora da qualidade de vida ${ }^{9,10}$, dois relatando a diminuição da necessidade de readmissão hospitalar ${ }^{11,12}$, um mostrou a melhora da mobilidade ${ }^{13}$ e outro demonstrou a redução do tempo de permanência hospitalar ${ }^{14}$.

Um estudo não mostrou diferença estatística significativa ou significância clínica diretamente relacionada intervenção do fisioterapeuta em $\mathrm{UE}^{13}$. A Tabela 2 mostra os principais ensaios clínicos sobre o assunto e seus resultados.

Tabela 2. Dados gerais sobre os ensaios clínicos incluídos

\begin{tabular}{|c|c|c|c|c|c|}
\hline $\begin{array}{c}\text { Autor/ } \\
\text { Ano }\end{array}$ & Amostre & Idede média & Objefivo & Intervenção fisioterapêutica & Resultedos \\
\hline $\begin{array}{l}\text { Lau ef al.r } \\
2008\end{array}$ & 121 & $50 \pm 19$ & $\begin{array}{l}\text { Avaliar o impacto da fisioterapia } \\
\text { na redução do quadro álgico em } \\
\text { pacientes com dor lombar aguda }\end{array}$ & $\begin{array}{l}\text { Educação, gestão da dor } \\
\text { através de exercícios } \\
\text { orientados, treinamento e } \\
\text { auxílio para caminhar }\end{array}$ & $\begin{array}{c}\text { Redução da intensidade da dor } \\
\text { e aumento da satisfação dos } \\
\text { pacientes }\end{array}$ \\
\hline
\end{tabular}

\begin{tabular}{|c|c|c|c|c|c|}
\hline $\begin{array}{l}\text { Calthorpe } \\
\text { ef al.r } \\
2014 \quad\end{array}$ & 90 & $56,2 \pm 21,3$ & $\begin{array}{l}\text { Avaliar os efeitos de um protocolo } \\
\text { de fisioterapia na mobilidade dos } \\
\text { pacientes de trauma }\end{array}$ & $\begin{array}{c}\text { Exercícios de balanço e força, } \\
\text { sedestação e deambulação } \\
\text { precoce }\end{array}$ & $\begin{array}{l}\text { Melhora da mobilidade no } \\
\text { terceiro e quinto dia após a } \\
\text { internação, porém nấo houve } \\
\text { impacto sobre o tempo de } \\
\text { estadia hospitalar ou } \\
\text { qualidade de vida. }\end{array}$ \\
\hline $\begin{array}{l}\text { Courtnet et } \\
\text { al., } 2009\end{array}$ & 128 & $78,8 \pm 6,9$ & $\begin{array}{l}\text { Avaliar o impacto da fisioterapia } \\
\text { sobre o risco de readmissão } \\
\text { hospitalar em pacientes idosos }\end{array}$ & $\begin{array}{l}\text { Programa de exercícios como } \\
\text { treino de equilíbrio, força e } \\
\text { marcha iniciando no hospital e } \\
\text { continuando por } 24 \text { semanas } \\
\text { após a alta }\end{array}$ & $\begin{array}{l}\text { Redução da necessidade de } \\
\text { readmissão hospitalar e } \\
\text { ofimização do SF-36 }\end{array}$ \\
\hline $\begin{array}{l}\text { Jesudason } \\
\text { ef al., } \\
2012 \quad\end{array}$ & 186 & $70,1 \pm 18,3$ & $\begin{array}{l}\text { Investigar o impacto de um } \\
\text { programa de fisioterapia sobre a } \\
\text { taxa de readmissão hospitalar }\end{array}$ & $\begin{array}{l}\text { Educação, aconselhamento, } \\
\text { treino de mobilidade, } \\
\text { prescrição de exercícios e de } \\
\text { recursos comunitários } \\
\text { apropriados }\end{array}$ & $\begin{array}{l}\text { Redução da taxa de } \\
\text { readmissão hospitalar no grupo } \\
\text { que realizou o programa de } \\
\text { fisioterapia. }\end{array}$ \\
\hline $\begin{array}{l}\text { Taylor et } \\
\text { al., } 2011\end{array}$ & 315 & $33 \pm 19$ & $\begin{array}{c}\text { Avaliar se a presença do } \\
\text { fisioterapeuta na unidade diminui o } \\
\text { tempo de permanência hospitalar } \\
\text { em pacientes com lesấo } \\
\text { musculoesquelética }\end{array}$ & $\begin{array}{c}\text { Foram realizados } \\
\text { alongamentos, cinesioterapia, } \\
\text { mobilização articular e } \\
\text { orientações }\end{array}$ & $\begin{array}{l}\text { Redução do tempo de } \\
\text { permanência hospitalar }\end{array}$ \\
\hline
\end{tabular}

\section{DISCUSSÃO}

Com base nos achados dos ensaios clínicos randomizados o fisioterapeuta tem um papel central em UE principalmente em relação a diminuição de quadro álgico, melhora da satisfação do paciente e diminuição da necessidade de readmissão hospitalar ${ }^{9-14}$.

Recente estudo afirma que existe uma necessidade de inovação na maioria das UE no mundo e que uma alternativa é a inclusão do fisioterapeuta nesses serviços. Nesse mesmo trabalho os autores verificaram que $89 \%$ dos pacientes que adentravam essa unidade tinham condições não graves, diagnóstico pelo médico da emergência, e que o retorno para as unidades primárias também foi em grande proporção, aproximadamente $46 \%{ }^{15}$. Ainda nesse trabalho, quarenta e seis por cento dos pacientes atendidos foram geridos de forma independente, sem qualquer apoio da equipe médica sendo assim a aplicação de procedimentos fisioterapêuticos avançados foram significativamente eficientes ${ }^{15}$. 
Crane e Delany ${ }^{16}$ avaliaram as responsabilidades e discussões éticas sobre a presença do fisioterapeuta na $U E$, revelando que existe ainda uma incerteza sobre a regulamentação e destacam a necessidade de uma educação para esclarecer e apoiar esta área de prática clínica, baseado em evidências clinicas apropriadas.

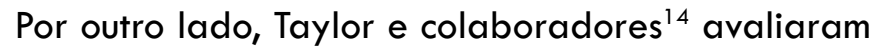
a influência da inserção do fisioterapeuta nas UE sobre o tempo de internação hospitalar em pacientes com lesões musculoesqueléticas periféricas, verificando uma redução do tempo de permanência desse perfil de paciente sem presença de efeitos adversos quando comparado ao atendimento secundário que seria fora da UE. Em um ensaio clínico randomizado, Jesudason e colaboradores ${ }^{12}$ avaliaram 186 pacientes e afirmaram que a fisioterapia na emergência reduz frequência de admissão hospitalar, porém não esteve associado com a frequência de reaparecimento ou retorno as atividades diárias.

A atuação do fisioterapeuta em UE é bastante ampla e no estudo de Lau e colaboradores ${ }^{9}$ foi avaliado o impacto da intervenção da fisioterapia em 121 pacientes com dor lombar. Nesse trabalho foi comparado o atendimento convencional com a abordagem fisioterápica especifica através de educação postural e treino para caminhada sendo assim nesse cenário houve uma diminuição significativa da dor e aumento da satisfação dos pacientes.

Porém, Calthorpe e colaboradores ${ }^{13}$ não verificaram melhora da qualidade de vida e necessidade de admissão hospitalar em pacientes admitidos na emergência. A justificativa para isso pode estar no perfil dos pacientes admitidos visto que nesse trabalho foram pacientes de trauma diferente do estudo anterior que eram pacientes com lombalgia. $\mathrm{Na}$ contramão do estudo de Calthorpe e colaboradores $^{13}$, Courtney e colaboradores ${ }^{11}$ verificaram melhora da qualidade de vida avaliada através do SF-12, principalmente no componente físico e mental. Foram estudados 128 idosos sobre um programa individualizado de fisioterapia. Outro desfecho encontrado foi a redução da necessidade de internamento hospitalar nessa população.

Já Richardson e colaboradores ${ }^{10}$ analisaram dois desfechos: satisfação do paciente e impacto da inserção da fisioterapia sobre os custos hospitalares. Ao estudarem 766 pacientes randomizados em dois grupos verificaram aumento da satisfação, porém um impacto não significativo sobre os custos e despesas hospitalares.

\section{CONSIDERAÇÕES FINAIS}

Com base no que foi visto nos artigos inclusos nessa revisão pode-se concluir que o fisioterapeuta tem um papel fundamental em UE principalmente no que se refere a diminuição do quadro álgico e redução da necessidade de readmissão hospitalar ou tempo de permanência no hospital. A capacidade de agregar trabalho, a possibilidade de discussão de casos com a equipe interdisciplinar e o cuidado integral e humanizado com o paciente e a família refletem a necessidade de inserção do profissional nas emergências.

\section{CONTRIBUIÇÕES DOS AUTORES}

Cordeiro ALL participou da concepção e desenho da pesquisa, obtenção de dados, análise e interpretação dos dados, análise estatística e revisão crítica do manuscrito. Lima TG participou da concepção e desenho da pesquisa, redação do manuscrito e revisão crítica do manuscrito.

\section{CONFLITOS DE INTERESSES}

Nenhum conflito financeiro, legal ou político envolvendo terceiros (governo, empresas e fundações privadas, etc.) foi declarado para nenhum aspecto do trabalho submetido (incluindo mas não limitandose a subvenções e financiamentos, conselho consultivo, desenho de estudo, preparação de manuscrito, análise estatística, etc).

\section{REFERÊNCIAS}

1. Neves $C A B$. Urgências e emergências em saúde: Perspectivas de profissionais e usuários. Cad Saúde Pública. 2006;22(3):691-4. doi: 10.1590/S0102$311 \times 2006000300024$

2. Cordeiro ALL. Atuação fisioterapêutica na unidade de emergência. In: Associação Brasileira de Fisioterapia Cardiorrespiratória e Fisioterapia em Terapia Intensiva; 
Martins JA, Andrade FMD, Dias CM, organizadores. PROFISIO Programa de Atualização em Fisioterapia em Terapia Intensiva Adulto: Ciclo 5. Porto Alegre: Artmed Panamericana; 2014. p. 77-108.

3. Ogawa KYL, Diniz JS, Frigeri LB, Ferreira CAS. Intervenção fisioterapêutica em emergências cardiorrespiratórias. $O$ Mundo da Saúde. 2009;33(4):457-66.

4. Piccoli A, Werle RW, Kutchak F, Rieder MM. Indicações para Inserção do Profissional Fisioterapeuta em uma Unidade de Emergência. ASSOBRAFIR Ciência. 2013;4(1):33-41.

5. Young SE, Bothwell JD, Walsh RM. Safely Managing Acute Osteoarthritis in the Emergency Department: An EvidenceBased Review. J Emerg Med. 2016;51(6):648-657. doi: 10.1016/i.jemermed.2016.05.052

6. Bodilsen AC, Klausen HH, Petersen J, Beyer N, Andersen $\mathrm{O}$, Jorgensen LM et al. Prediction of Mobility Limitations after Hospitalization in Older Medical Patients by Simple Measures of Physical Performance Obtained at Admission to the Emergency Department. PLoS One. 2016;19;1 1(5):e0154350. doi: 10.1371/iournal. pone. 0154350

7. Oliveira EB, Lisboa MTL, Lúcido VA, Sisnando SD. A inserção do acadêmico de enfermagem em uma unidade de emergência: A psicodinâmica do trabalho. Revista Enfermagem UERJ. 2004;1 2:179-185.

8. Olivo SA, Macedo LG, Gadotti IN, Fuentes J, Stanton $T$ and Magee DJ. Scales to assess the quality of randomized controlled trials: A systematic review. Phys Ther 2008;88(2):156-175. doi: 10.2522/pti.20070147

9. Lau PM, Chow DH, Pope MH. Early physiotherapy intervention in an Accident and Emergency Department reduces pain and improves satisfaction for patients with acute low back pain: a randomised trial. Austrilian Journal of Physiotherapy. 2008;54(4):243-249.

10. Richardson B, Shepstone L, Poland F, Mugford M, Finlayson $B$, Clemence N. Randomised controlled trial and cost consequences study comparing initial physiotherapy assessment and management with routine practice for selected patients in an accident and emergency department of an acute hospital. Emerg Med J. 2005;22(2):87-92. doi: $10.1136 / \mathrm{emi} .2003 .012294$

11. Courtney M, Edwards H, Chang A, Parker A, Finlayson $K$, Hamilton K. Fewer Emergency Readmissions and Better Quality of Life for Older Adults at Risk of Hospital Readmission: A Randomized Controlled Trial to Determine the Effectiveness of a 24-Week Exercise and Telephone FollowUp Program. J Am Geriatr Soc. 2009;57:395-402.

12. Jesudason $C$, Stiller K, Mclnnes M, Sullivan T. A physiotherapy service to an emergency extended care unit does not decrease admission rates to hospital: a randomised trial. Emerg Med J. 2012;29:664e669.
13. Calthorpe S, Barber EA, Holland AE, Kimmel L, Webb MJ, Hodgson C et al. An intensive physiotherapy program improves mobility for trauma patients. J Trauma Acute Care Surg. 2014;76(1):101-106. doi: 10.1097/ TA.0b013e3182ab07c5

14. Taylor NF, Norman E, Roddy L, Tang C, Pagram A, Hearn K. Primary contact physiotherapy in emergency departments can reduce length of stay for patients with peripheral musculoskeletal injuries compared with secondary contact physiotherapy: a prospective non-randomised controlled trial. Phyisiotherapy. 97(2):107-114.

15. De gruchy A, Granger C, Gorelik A. Physical Therapists as Primary Practioners in the Emergency Department: Six-Month Prospective Practice Analysis. Physical Therapy. 2015;95(9): 1 207-61. doi: 10.2522/pti.20130552

16. Crane J, Delany C. Physiotherapists in emergency departments: responsibilities, accountability and education. Physiotherapy. 2013;99(2):95-100. doi: 10.1016/i. physio.2012.05.003 